\title{
Shining light on the brain to understand how it works and to help children who face difficulties
}

\author{
Laura Bell 1, Vanessa Reindl ${ }^{1,2}$, Jana A. Kruppa ${ }^{1,2}$, Alexandra Niephaus ${ }^{1}$, Simon H. Kohl 1,2, \\ \& Kerstin Konrad 1,2,*
}

${ }^{1}$ Child Neuropsychology Section, Department of Child and Adolescent Psychiatry, Psychosomatics and Psychotherapy, Medical Faculty, RWTH Aachen University, D-52074 Aachen, Germany.

${ }^{2}$ JARA-Brain Institute II, Molecular Neuroscience and Neuroimaging (INM-11), RWTH Aachen \& Research Centre Juelich, D-52428 Juelich, Germany.

* Correspondence:

k.konrad@fz-juelich.de

Keywords: fNIRS; functional near-infrared spectroscopy; light; brain; brain activation;

\begin{abstract}
Have you ever thought that light could tell you something about your brain? Light is a powerful tool that helps brain researchers understand the brain. Our eyes can only see less than $1 \%$ of the total light around us. Some of the light is red, so-called near-infrared light. It can run through your head and the top layers of your brain and thereby gains important information about your brain activation. The technique that uses near-infrared light is called functional near-infrared spectroscopy. This term is very long, so we will call it "fNIRS" from now on. In this article, we will first show you how a fNIRS machine looks like and what it is like to take part in a fNIRS experiment. Next, we will explain how we can use near-infrared light to better understand the brain. Finally, we will give you some examples of what fNIRS can be used for and how we can use it to help children who face difficulties in their daily lives.
\end{abstract}




\section{Using light to see in the dark}

Imagine you want to find out what's hidden in a dark treasure chamber. You cannot see anything due to the darkness. To see something, you would probably reach for a flashlight to help you discover what is hiding in the dark. This is actually a strategy that brain researchers use in a similar way to see what is going on in a person's brain. The brain is a dark black box just like the dark treasure chamber. To see something within this black box, brain researchers use a technology that is called functional nearinfrared spectroscopy [1]. That's a very complicated and long word. Brain researchers often simply call it "fNIRS". We will use the word fNIRS now as well! As you can see in Figure 1, the fNIRS machine looks like a computer. Cables are connected to this computer with colorful plugs at their end. These plugs are the sensors of the machine and are called optodes (optical sensors). The red sensors or senders send light (like flashlights lighting up the brain) and the blue sensors or receivers receive light (like eyes seeing what is going on in the brain). The light and the sensors help brain researchers to see what happens in your brain.

To investigate what is going on in the brain, brain researchers set up different experiments. These are carefully designed tests or tasks during which you are connected to a fNIRS machine. When you take part in a fNIRS experiment, the sensors are placed onto your head. Because they must touch the skin of your head, the underlying hair is first brushed aside. Moreover, they must sit still on your head otherwise the signal may be blurred. To make sure that the sensors do not move, they are often inserted into a cap or fixed with a band around your head. Then you are asked to do things using your brain, for example solving math, reading a book, or playing a game. At the same time, the fNIRS machine records what happens in your brain while you are doing your task. In Figure 1, you can see what it looks like when you are connected to a fNIRS machine.

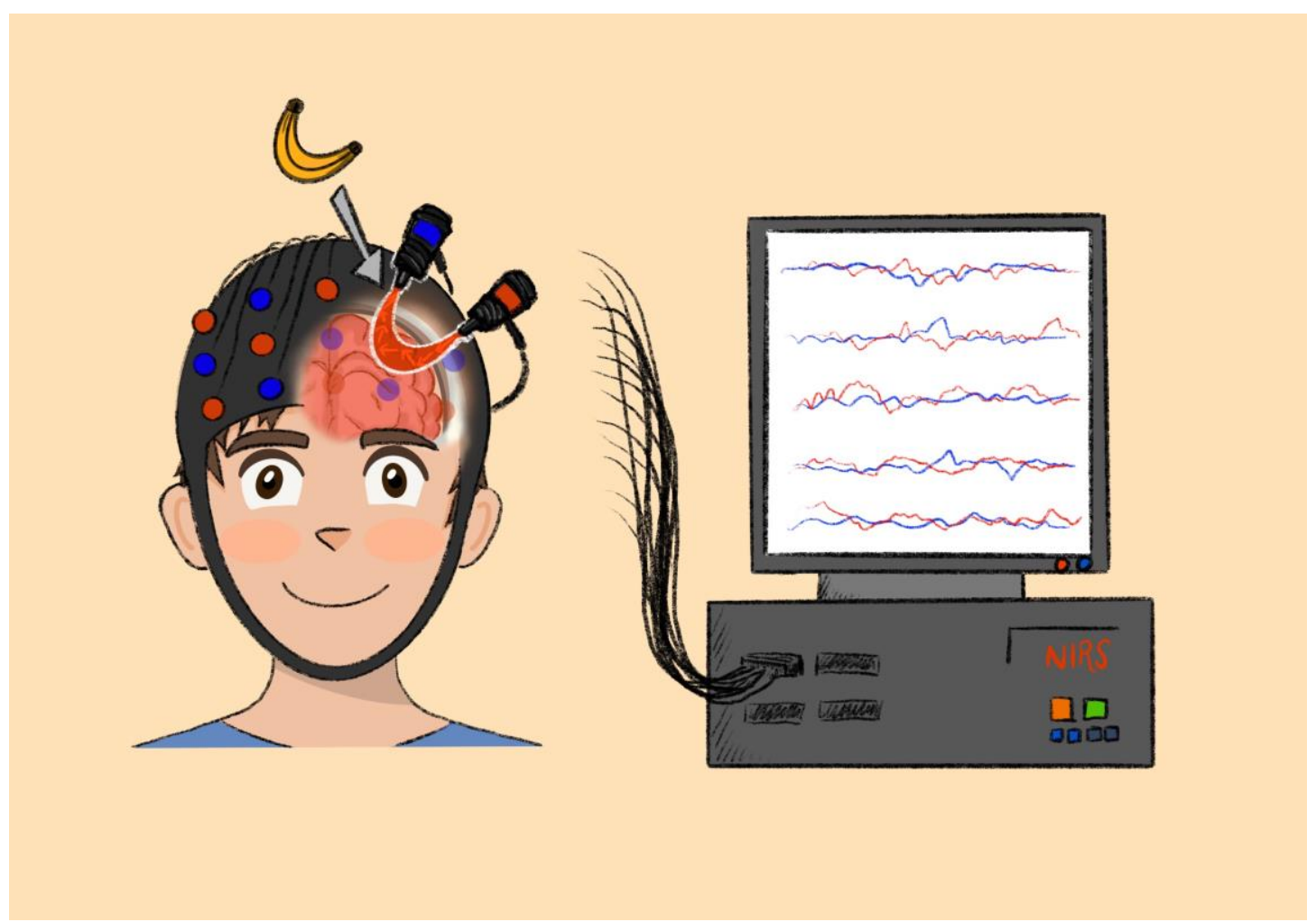


Figure 1 - What does a fNIRS machine look like and how can it see what your brain does? The picture above shows a boy who is connected to a fNIRS machine. You can see red and blue fNIRS sensors, some send light onto the head, the red ones, and some receive light, the blue ones. You can also see how the near-infrared light travels through the hair, skin, skull and the brain in a banana-like shape. The red and blue wiggly lines on the screen of the fNIRS machine show the current amount of light. The machine works this out by subtracting the amount of light the blue sensors receive from the amount the red sensors sent.

\section{How do we know that light can tell us something about the brain?}

This dates back to a discovery of Fransis Jobsis in the year 1977. At dinner with his family, he was astonished to observe that it is possible to shine red light through a 3- or 4-mm thick bone of a beefsteak, while he was holding it against the light. But why is that? If you look at a rainbow you can see that light can appear in many different colors, such as violet, blue, green, yellow or red. This is because the light is broken up into its components, the different wavelengths, each representing one color. Have you ever been swimming in the ocean? A wavelength is the distance between the highest points, two peaks, of a light wave, similar to the distance between waves in the ocean. So, wait! Light components are traveling in different kinds of waves? Scientists discovered that when you shine a light upon an object, the light travels in alternating light and dark rings - sort of wave-like. Some colors are travelling in large, some in small waves (see Figure 2). It's like when you throw a pebble in a pond. Some waves are larger than others. All light waves travel together along a straight line through the air, that is what we call a ray of light - just like the sun rays! The red light that Fransis Jobis used to shine through the bone is the same light a fNIRS machine uses. It has a wavelength of between 650 and 850 nanometers. A nanometer comes from the Greek word for "dwarf" and it is small. It is really small. In fact, it is so small you can't even see one with your own eyes! You can get 10 million of them in just 1 centimeter, and even your hair's thickness is probably 80,000 - 100,000 nanometers! The nanometer range of the light that fNIRS uses is called the "near-infrared spectrum" and that is where the name of the technology comes from, near-infrared spectroscopy. Near-infrared light is harmless to the body. It is able to shine through biological tissue, like Fransis' beefsteak or your brain, quite well. Due to this property, it provides us with a unique window to look into the human brain, which we often call the "optical window" (Figure 2).

When placed on the head, the near-infrared light travels from the red sensors (the senders) through several layers, including our skin, the skull, and the protective fluid surrounding the brain, until the light reaches the brain. The path of the light follows a banana-like shape back to the surface of the head where the blue sensors (the receivers) are placed (Figure 1). The receivers then measure how much of the red light returns to the surface of the head. On its journey through the different layers, some of the light is scattered. Just like when using your flashlight in the dark treasure chamber, some of the light will fall onto objects and will change its paths, while some light travels straight in the direction that you hold the flashlight. This is called scattering. As well as scattering, some light is swallowed, or absorbed, by the objects in the chamber. In the case of the brain, light is absorbed by the particles in the body, such as water, fat or blood particles. Because of scattering and absorption, not all of the light which is sent through the brain returns to the receivers.

But how exactly can brain researchers then use near-infrared light to look at the brain? Within the optical window, light is mainly absorbed by a protein in the blood, called hemoglobin. Hemoglobin makes your blood red! But hang on! This will become important later. 


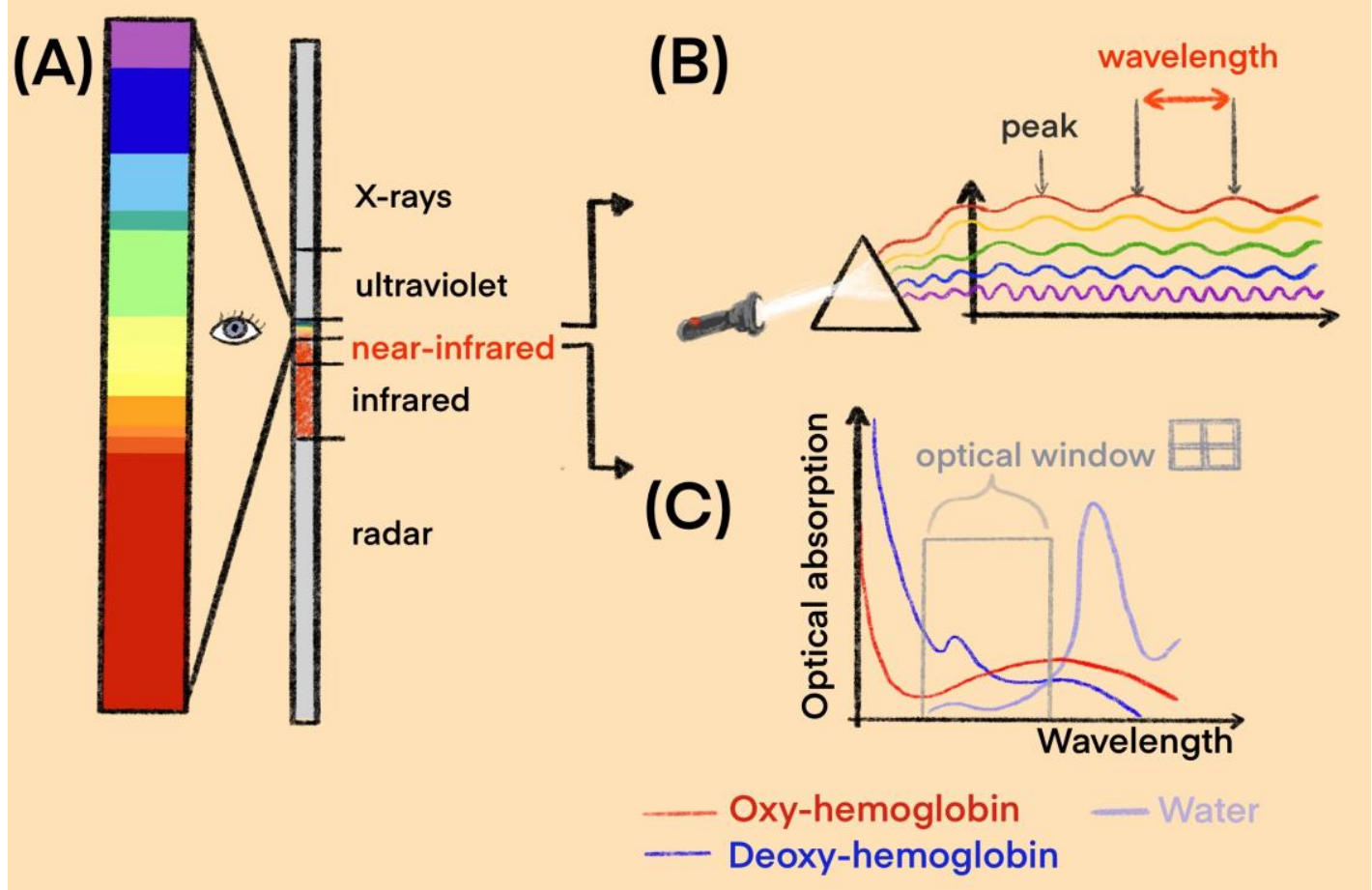

Figure 2 - What kind of light does the fNIRS machine use and what is the "optical window"? (A) There are different kinds of light. Some, but not all, are visible to our eyes. (B) Each light has a specific wavelength. A wavelength describes the distance between two peaks, the highest points, of a wave. (C) fNIRS uses near-infrared light, which is able to shine through biological tissue like our brain. Therefore, the light-range that fNIRS machines use is often referred to as the optical window that allows brain researchers to look into the brain.

\section{How does the light tell us which part of your brain is active?}

The brain consists of more than 100 billion cells, called neurons. Like yourself, these neurons need to eat and breath. When the neurons in the brain become active, the body starts sending an extra amount of blood to those parts of the brain that are active. This mechanism is called the hemodynamic response (see [2] for a comparable explanation of the hemodynamic response and how it can be similarly measured with a technique that is called magnetic resonance imaging). So, the brain is a very clever organ: More blood means more energy and more oxygen, which makes your brain perform better. So, it is very similar to what happens when you are exercising and doing sports: During exercise, your body uses more oxygen and you breathe deeper and more often to take in the oxygen needed. The increased breathing delivers more oxygen into the blood stream, which is then transported to your working muscles.

Similarly, when you think, certain parts of your brain become active and these regions of the brain have blood that contains more oxygen. Oxygen is carried by the protein hemoglobin. Hemoglobin that carries oxygen is called oxy-hemoglobin, while hemoglobin without oxygen is called deoxyhemoglobin. When the neurons become active, they use up oxygen. At the same time, they tell the 
blood vessels to become wider so that more blood can flow to the activated brain area. This increased blood flow brings more oxygen (and hemoglobin) to the activated brain area than the neurons actually use. As a result, the amount of oxy-hemoglobin increases and deoxy-hemoglobin decreases (Figure 3). These changes are measured by fNIRS. When the amount of oxy- and deoxy-hemoglobin in a brain region changes, more or less light is absorbed when traveling through this brain area. Why is that?

You may have noticed that blood is red, but can have slightly different red colors. The larger blood vessels that you can sometimes see through the skin of your arm may even appear blueish. This is due to the amount of oxygen in your blood (oxy- and deoxy-hemoglobin). Oxygen-rich blood (oxyhemoglobin) has a bright red color and oxygen-poor blood (deoxy-hemoglobin) has a darker red purple color. Hemoglobin absorbs the light, which is sent by the fNIRS machine. Now if your brain becomes more active and needs more oxygen, the increased amount of oxygen and hemoglobin in your blood stream takes up more of the light than deoxy-hemoglobin does. Therefore, the amount of light on its journey back to the blue receivers is reduced.

Based on the difference between how much light is send by the red senders and received by the blue receivers, brain researchers can calculate changes in oxy- and deoxy-hemoglobin. That means, they know which brain region is involved in which task. That's a cool "trick", don't you think? It is called inference. Brain researchers do not measure exactly the brain's activity but infer it (get this information) from light and the amount of oxy-hemoglobin. Thus, more oxy-hemoglobin in a given brain area means more brain activation in that same area!

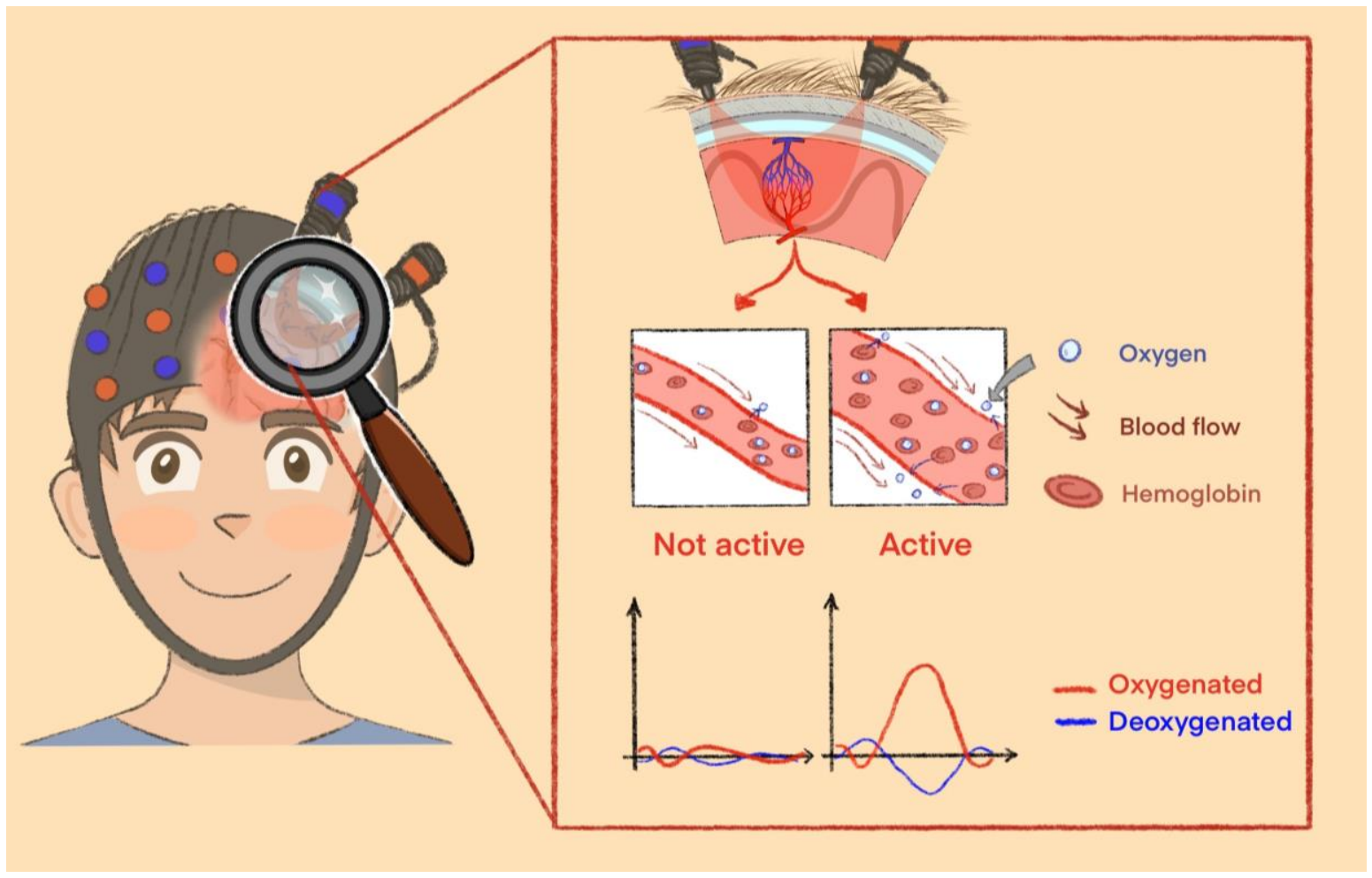

Figure 3 - How can light see what the brain does?

To make sure that a brain region works well, the blood vessels in the brain have to transport oxygen to each brain region. Oxygen travels within the blood vessels with the help of a protein that is called hemoglobin. When a brain region is active, it needs more oxygen than during rest. To increase the 
oxygen in the active brain area, blood vessels become wider and the blood flows faster. Thereby, more oxygen can be transported to the brain area where it is needed. These changes in oxygenated and deoxygenated hemoglobin concentrations within the blood vessels are measured with fNIRS.

But wait! fNIRS does not allow brain researchers to see everything within the brain. You may have noticed that when you use a flashlight in a dark room, you still can't see everything either. Probably, the objects closest to you will appear most clearly. Similarly, as the fNIRS sensors are placed on the outside of the head, brain researchers can only see what is going on in the outer surface of the brain, called the cerebral cortex.

\section{What can we use fNIRS for and how can we use it to help children who are facing difficulties?}

Our brains support us in all of our activities in everyday life. There is no single activity that we do NOT need our brains for. Even during sleep! Can you imagine that? Simply by reading this article, regions in your brain have been activated at this very moment. If you were now connected to a fNIRS machine, we could see which areas of your brain are involved in reading. Thus, fNIRS machines can record almost all of our daily activities, in order to support us brain researchers to find out which brain areas are needed for each activity. It can be used for research in children and even babies. Importantly, children who are facing difficulties, for example with attention or reading, can also take part in a fNIRS study. Similarly, we can use it to investigate the brains of children that have trouble hearing [3] or difficulties moving or making friends and social contact [4]. Brain researchers are interested in understanding why the brains of these children react differently in certain situations. This might help the brain researchers to understand them better and develop better treatments to help them. FNIRS might even help us to train the brains of these children to help them improve. This is called a neurofeedback training [5] and involves measuring your brain activity and showing it to you live on a computer screen. You then try to activate your brain a little more with your thoughts and imagination and see on the screen if you are successful. It is like a game that you play with your brain: you win when you activate your brain! Figure 4 shows some of the examples how fNIRS can be used for.

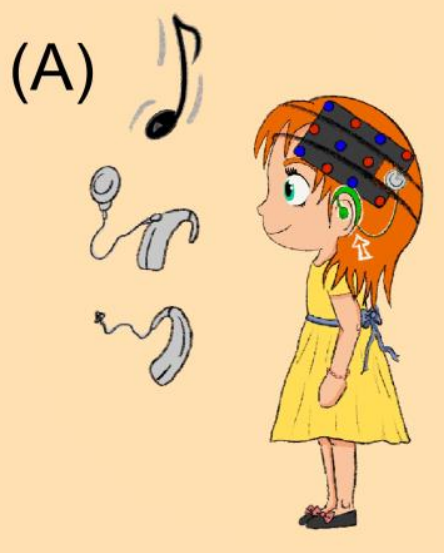

(B)

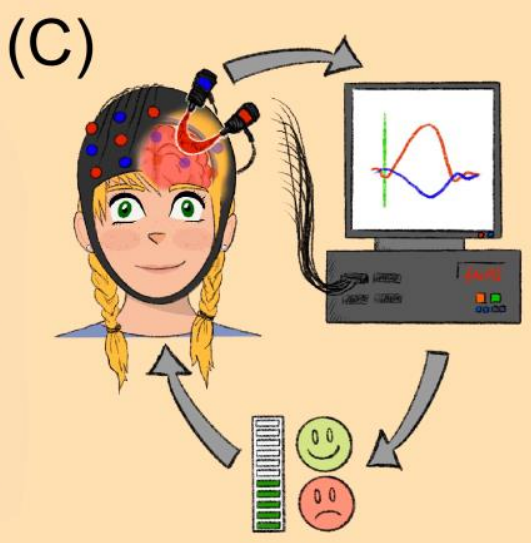


Figure 4 - Examples of numerous possibilities to use fNIRS to help children with difficulties. fNIRS can be used to examine the brains of children who face difficulties with attention and hearing (A), with making friends and social contact (e.g., by measuring two children at the same time) (B), and many more situations. In addition, we might even use it to train the brains of children with neurofeedback (C).

If you would like to find out more about the advantages and limitations of fNIRS you can read the recently published article by Soltanlou \& Artemenko [6]. They describe how fNIRS can be used to understand how the brain works in the classroom and during many more activities!

If you would like to see a video about the way fNIRS works and some examples of what we can use fNIRS for, click on the following link:

https://youtu.be/RE_dyHxtKiI

So, fNIRS offers many opportunities to understand the brain. We are excited to see what else we can find out about the brain! Maybe you will join us one day? 


\section{Glossary}

Hemodynamic response - The mechanism of your body that regulate your blood flow to ensure that a brain region gets enough fuel (oxygen and glucose) to function.

Hemoglobin - A protein in your blood that transports oxygen to different parts of the body.

fNIRS - functional near-infrared spectroscopy, a technique that brain researchers use to see whether certain areas of your brain are active.

Neurons - Specialized nerve cells in the brain that are linked with each other to communicate. Neurons need more oxygen in active parts of the brain.

Optode - A measurement sensor that uses optical information, for example near-infrared light. Optodes can either send (the sender) or receive (the receiver) light.

\section{References}

[1] Pinti, P., Tachtsidis, I., Hamilton, A., Hirsch, J., Aichelburg, C., Gilbert, S., \& Burgess, P. W. 2020. The present and future use of functional near-infrared spectroscopy (fNIRS) for cognitive neuroscience. Annals of the New York Academy of Sciences. 1464.1: 5.

[2] Hoyos, P. M., Kim, N. Y., \& Kastner, S. 2019. How Is Magnetic Resonance Imaging Used to Learn About the Brain? Frontiers for Young Minds. 7:86. doi: 10.3389/frym.2019.00086

[3] Bell, L., Scharke, W., Reindl, V., Fels, J., Neuschaefer-Rube, C., \& Konrad, K. 2020. Auditory and Visual Response Inhibition in Children with Bilateral Hearing Aids and Children with ADHD. Brain Sciences. 10.5: 307.

[4] Kruppa, J. A., Reindl, V., Gerloff, C., Weiss, E. O., Prinz, J., Herpertz-Dahlmann, B., ... \& Schulte-Rüther, M. (2020). Brain and motor synchrony in children and adolescents with ASD-an fNIRS hyperscanning study. Social Cognitive and Affective Neuroscience.

[5] Bado, P., Stewart, M., \& Moll, J. 2016. Training Your Emotional Brain: From Science Fiction to Neuroscience. Frontiers for Young Minds. 4:21. doi: 10.3389/frym.2016.00021

[6] Soltanlou, M., \& Artemenko, C. 2020. Using Light to Understand How the Brain Works in the Classroom. Frontiers for Young Minds. 8:88. doi: 10.3389/frym.2020.00088 


\section{Author Contributions}

LB, VR, JAK, AN, and SHK wrote the manuscript together. KK revised the manuscript critically. LB created the illustrations. All authors contributed to the conception and design of the manuscript. All authors have read and approved the final version for publication.

\section{Conflict of Interest}

SHK receives payments to consult with the start-up company Mendi innovations AB, Stockholm, Sweden.

LB creates paid illustrations for NIRx Medizintechnik GmbH, Berlin, Germany.

The remaining authors declare that the research was conducted in the absence of any commercial or financial relationships that could be construed as a potential conflict of interest.

\section{Acknowledgements}

We would like to thank all children and adolescents who stimulated the writing of the current manuscript by asking interesting questions about our brains and how brain imaging works.

The fNIRS machine described here was purchased by the University Hospital RWTH Aachen (Germany) and funding was supported by a grant of the German Research Foundation (DFG) (INST 948/18-1 FUGG) awarded to KK. 


\section{Author biography and photo of each author}

\section{Laura Bell, M.Sc.}

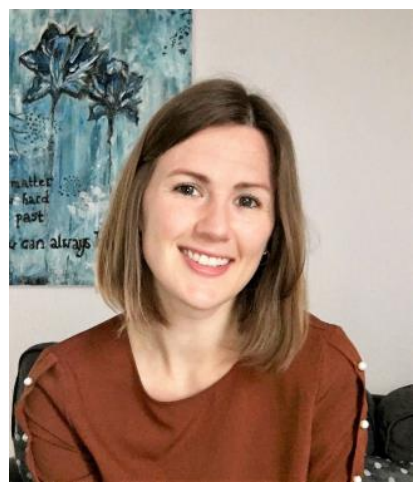

I am a brain researcher that conducts studies with fNIRS and is interested in multisensory processing, its development and auditory deprivation. In other words, I am fascinated by how the brain learns to process what we see, hear, smell or feel and how the brain can adjust itself when we lose one sense such as hearing. Isn't it crazy that the brain tries to compensate for a lost sense and can even use the input from external listening devices such as cochlear implants and hearing aids? Besides research, I love being outside, doing sports and drawing - for example illustrations for this article.

\section{Vanessa Reindl, PhD}

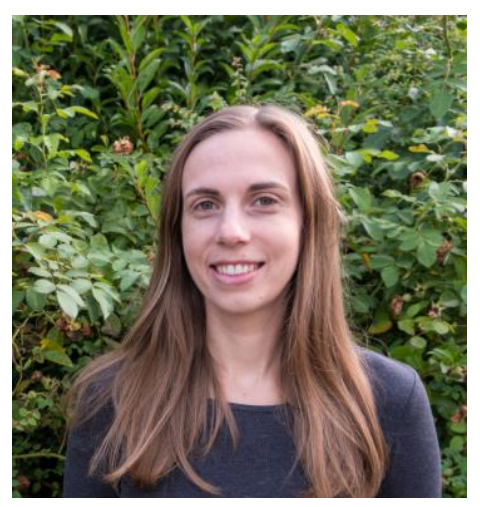

In my research I study what happens in the brain and in the body when parent and child play with each other, using methods like functional near-infrared spectroscopy. To investigate this, we measure the brain activities of both people at the same time. It is fascinating to see that when we interact or communicate with each other, our brain activities are in sync, just as you may synchronize your footsteps when you walk next to your friend. Besides research, I enjoy spending time outdoors, going jogging, hiking or skiing in the winter. 
Jana A. Kruppa, M.Sc.

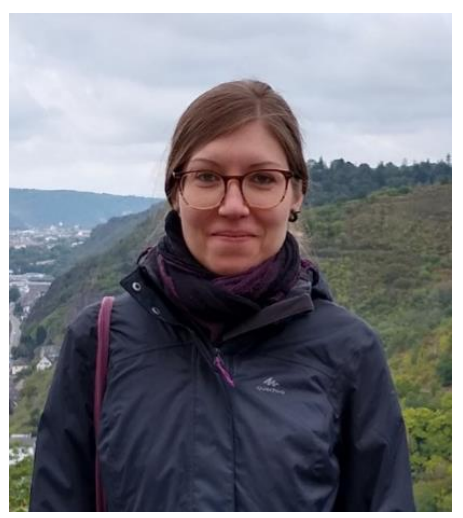

In my work as a brain researcher, I investigate brain activity and behavior of children, adolescents, and young adults who encounter difficulties in their everyday life. For example, they may struggle to interact or communicate with others or to concentrate for a longer period of time. With my work, I hope to contribute to the development of better treatment methods helping these individuals feel better. Besides research, I enjoy spending time with friends, doing Yoga, and riding my road bike. I love animals, spending time with my two cats and being in nature.

\section{Alex Niephaus, M.Sc.}

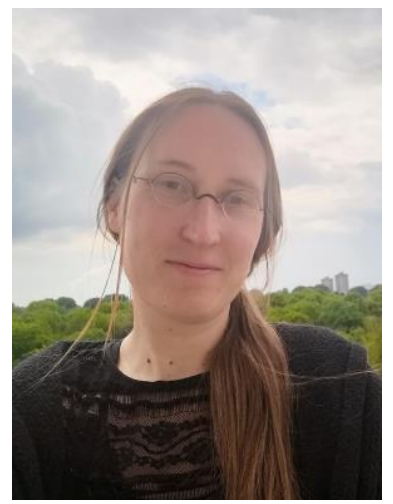

I'm a brain researcher and also a speech and language therapist. Certainly, this means that I'm very interested in brain function during communication. fNIRS is a perfect tool for that because you can not only investigate people in situations close to everyday life but also measure more than one person at the same time (hyperscanning). Mostly, I'm researching the brain activations of people who stutter or whose speech is way too fast and bumpy and therefor hard to understand (cluttering). Apart from doing brain research, I really enjoy playing adventure games on the computer or playing card and board games with my friends. 
Simon H. Kohl, M.Sc.

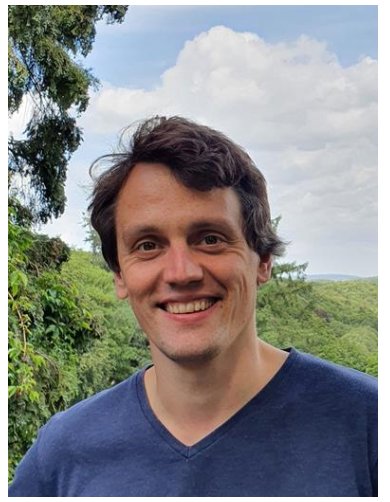

I am a psychologist and neuroscientist. I am very fascinated by neurofeedback, a form of brain training where people can look at their own brain activity in real-time and learn how to control it. How is the brain learning to regulate itself? Can we use neurofeedback to change brain functions and eventually improve people's lives? Or is it just a hype? For this purpose, I am currently working with fNIRS, which is a great tool because it is so easy to use. Besides neuroscience and psychology, I am a passionate martial artist, and love to spend my time outside and meet friends.

Kerstin Konrad, Uni-Prof. Dr. rer. nat.

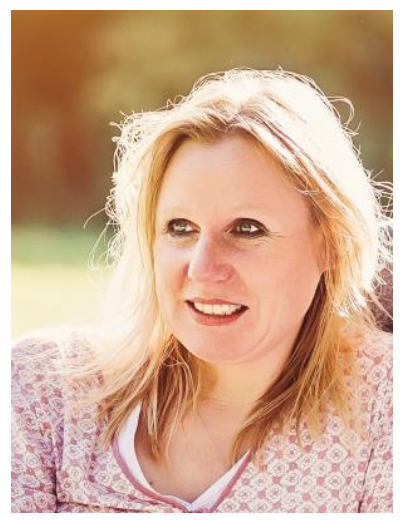

I am a professor in Clinical Child Neuropsychology at the RWTH Aachen University/Germany and the Research Center Juelich. I study how the brain develops from infancy to young adulthood and what happens if brain development goes awry. With my work, I hope to help children who experience difficulties at school, with their families or friends to feel better, to better concentrate and to improve their emotion regulation and well-being! I think that brain research with children and for children is the most fascinating job I can imagine. 